\title{
Effectiveness of Physical Exercise in Older Adults With Mild to Moderate Depression
}

\author{
Jesús López-Torres Hidalgo ${ }^{1,2}$ \\ Joseba Rabanales Sotos ${ }^{3}$ \\ DEP-EXERCISE Group*
}

${ }^{1}$ Albacete Zone VIII Health Center, Albacete, Spain

${ }^{2}$ Faculty of Medicine, University of Castile-La Mancha, Albacete, Spain

${ }^{3}$ Albacete Faculty of Nursing, University of Castile-La Mancha, Albacete, Spain

\begin{abstract}
PURPOSE We sought to compare the effectiveness of physical exercise with that of treatment with antidepressant drugs routinely used in clinical practice, in terms of decreasing depressive symptomatology in patients aged $\geq 65$ years who present with clinical criteria of a depressive episode.
\end{abstract}

METHODS We conducted a randomized clinical trial in a primary care setting. A total of 347 patients aged $\geq 65$ years with a clinically significant depressive episode were randomized to participation in a supervised physical exercise program or to receive antidepressant treatment by their general practitioners.

RESULTS Intention-to-treat analysis showed that the cumulative incidence of improvement in depressive symptomatology (Montgomery-Åsberg Depression Rating Scale score $<10$ ) in the physical activity (PA) group after 1 month was not significantly different from that in the antidepressant treatment (AT) group. However, the proportion of those who showed improvement was significantly greater $(P<.01)$ in the AT group $(60.6 \%$ and $49.7 \%)$ compared to the PA group $(45.6 \%$ and $32.9 \%)$ at the end of 3 and 6 months, respectively. The number of withdrawals was greater in the PA group (39.2\% and $58.2 \%$ ) compared to the AT group (22.6\% and $40.0 \%$ ) at 3 and 6 months, respectively, yet the proportion of participants with adverse side effects was greater in the AT group $(8.9 \%$ vs $22.5 \% ; P=.007)$.

CONCLUSION Although improvement was initially similar in both treatment groups, AT was superior in the medium term, despite giving rise to a greater number of adverse effects.

Ann Fam Med 2021;19:302-309. https://doi.org/10.1370/afm.2670.

\section{INTRODUCTION}

$\mathrm{D}$ epression is a common and disabling disorder that affects more than 120 million people worldwide and a minimum of 1 in every 5 people over the course of their lifetime. ${ }^{1}$ Although depression is routinely treated with antidepressant drugs and/or psychologic therapy, other therapeutic alternatives have received growing attention in recent years. Physical exercise in particular might be beneficial for depressed patients and equivalent to antidepressant treatment. ${ }^{2-4}$

Depressive disorders in older adults are characterized by considerable diagnostic complexity, a large degree of clinical polymorphism (difficulty in recognizing depressive symptoms, frequent somatic complaints, etc), and a high related risk of disability, with a potentially severe impact on quality of life. ${ }^{4}$ Old age can be a time of great emotional fragility; aside from neurobiologic changes during brain aging, there are important losses that affect older adults' emotions, physical condition, and social status. Depression is the most frequent psychiatric disorder among persons of advanced age, with $8 \%$ to $16 \%$ of all community-dwelling older adults presenting with clinically significant depressive symptoms. ${ }^{5}$

Antidepressant drugs often give rise to undesirable side effects, especially in the elderly population, with the use of such drugs often being prolonged indefinitely or unnecessarily. It seems reasonable, therefore, to 
test new therapeutic modalities that might result in fewer adverse side effects and lead to lower health care costs. Among the reasons why exercise might improve depression is the belief that it can act as a distraction from negative thoughts, and the fact that an important aspect of exercise might involve the acquisition of a new skill. In addition, social contact might form part of this mechanism, given that weak social support is broadly known to be a strong predictor of depressive symptomatology among community-dwelling older adults. ${ }^{6}$ Moreover, physical activity can have physiologic effects, such as changes in endorphin and monoamine levels or a decrease in the level of the stress hormone cortisol, which can improve mood. ${ }^{7}$ Some studies suggest that exercise stimulates the growth of new neurons as well as the release of proteins, such as brain-derived neurotrophic factor, which enhance the survival of neurons. ${ }^{8-9}$ Nonetheless, considerable uncertainty still surrounds the effectiveness of exercise for depression, ${ }^{10-11}$ owing mainly to methodologic considerations concerning the studies that have been carried out. ${ }^{12}$ It should be stressed that until now, older adults have been little represented in clinical trials in which both pharmacologic and nonpharmacologic measures for depressive disorders have been evaluated.

We sought to compare the effectiveness of physical exercise with that of treatment with antidepressant drugs routinely used in clinical practice, in terms of decreasing depressive symptomatology in patients aged $\geq 65$ years presenting with clinical criteria of a depressive episode diagnosed in primary care, over a 6 -month follow-up period. We hypothesized that supervised physical exercise of moderate intensity, combined with aerobic and resistance training, could be appropriate for decreasing depressive symptoms in older adults.

\section{METHODS}

We conducted a parallel trial with 2 randomized groups, using a previously published study protocol. ${ }^{13}$ Participants were selected at 10 family medicine outpatient clinics belonging to 3 health centers (Zones IV, VB, and VIII) in the city of Albacete, in southeast Spain. The target population comprised persons aged $\geq 65$ years, presenting with criteria of a clinically significant depressive episode (as defined by the International Classification of Diseases, 10th Revision). The inclusion criterion was defined as any patient affiliated with the participating health centers who had a mild to moderate depressive episode scoring at least 10 points on the Montgomery-Åsberg Depression Rating Scale (MADRS). ${ }^{14-15}$ The exclusion criteria were as follows: physical or mental limitation capable of preventing participation in the study, contraindication(s) for physical exercise, severe depressive disorder (important interference in social or occupational functioning, psychotic symptoms, or suicidal ideation), or use of antidepressant drugs. Initial physical aptitude was evaluated for all participants by reference to resting heart rate (measured with a heart rate monitor) and submaximal heart rate, calculated on the basis of maximum heart rate (220 minus age) on an indoor exercise bicycle $(70 \%-85 \%$ of maximum heart rate at a constant load and speed for 6 minutes).

After compliance with the inclusion criteria and consent to participate had been ascertained, patients were randomly and equiprobably allocated to the physical activity (PA) or antidepressant treatment (AT) group. Allocation was performed centrally at the Zone VIII Health Center using a computer-generated random sequence of numbers. All study patients provided written informed consent to participate in the trial. The study was approved by the Albacete Health Zone Clinical Research Ethics Committee and was conducted in full conformity with the Helsinki Declaration and Good Clinical Practice Guidelines.

Of the 432 patients who were preselected, 347 provided consent and were randomized to 1 of the 2 groups (176 to the PA group and 171 to the AT group) (Figure 1). Subsequently, 23 were excluded owing to the presence of exclusion criteria (severe depressive disorder or contraindication for inclusion in a PA program), and an additional 11 were excluded for withdrawing consent, resulting in a total of 313 patients included in the study (158 in the PA group and 155 in the AT group). Over the follow-up period, an additional 46 patients withdrew consent, and 120 dropped out for different reasons (including those who dropped out before starting treatment). A total of 66 patients in the PA group and 93 in the AT group completed follow-up.

To verify the hypothesis that physical exercise might be appropriate for decreasing depressive symptoms in older adults (null hypothesis: physical exercise is less effective than antidepressant treatment; alternative hypothesis: physical exercise is at least as effective as antidepressant treatment), a sample size of 313 patients was calculated. This sample size corresponded to an expected $75 \%$ positive response in both the PA and AT groups (a decrease in depressive symptomatology until registering a MADRS score $<10$, usually considered indicative of an absence of depressive disorder), an alpha risk of 0.025 , a beta risk of 0.20 , a noninferiority margin of $15 \%$ (maximum difference of response for AT to be deemed not inferior to supervised physical exercise), and a percentage loss to follow-up of $20 \%$. The recruitment period was from February 2017 to March 2019. 
Supervised physical exercise consisted of a physical activity program of two 1 -hour sessions per week over a period of 6 months (a total of 48 sessions in groups of 10-12 persons), run by a fully qualified sports instructor with a degree in physical activity and sports sciences and held at facilities belonging to the Albacete Zone VIII Health Center. The sessions included a balanced combination of aerobic, muscle strength, and flexibility exercises, as well as exercises designed to improve balance. The sessions also included educational content on how to increase physical effort in daily life and perform regular exercise adapted to individual age and fitness to maintain appropriate body weight. Over the course of the sessions, participants were trained to increase their level of physical activity and reach a point at which they would perform at least 30 minutes of moderately intense exercise every day. This intervention was implemented from a behavioral

\section{Figure 1. General study flow chart.}

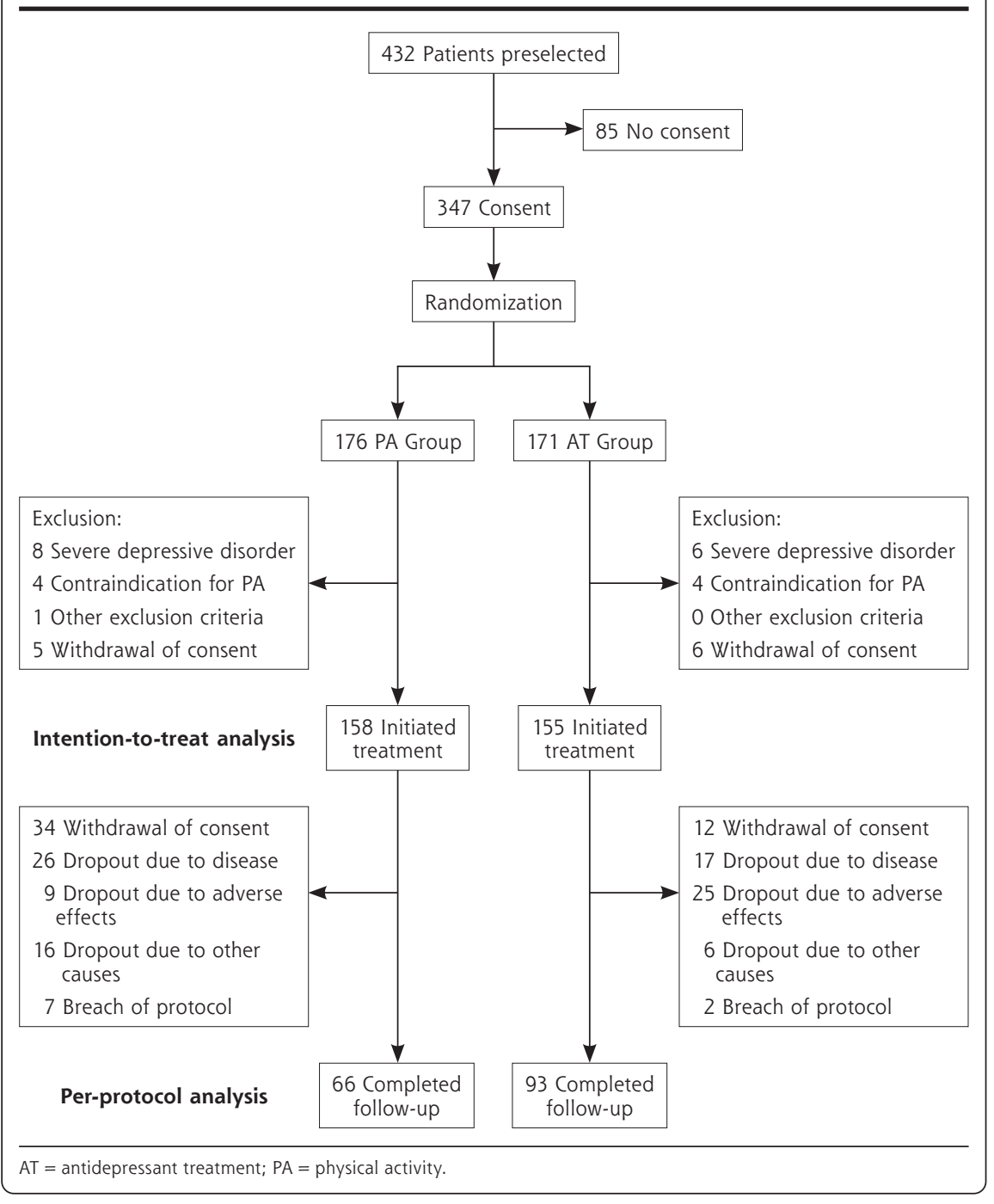

perspective, with a view to trying to motivate participants to change and improve their physical activity habits. With regard to the treatments prescribed in the AT group, each patient's general practitioner made the choice of the most appropriate drug, taking into account the patient's characteristics.

The duration of follow-up was 6 months, with evaluations at baseline and 1, 3, and 6 months. The principal outcome variable was decrease in depressive symptomatology, with a positive result defined as a MADRS score $<10$ points, which is indicative of an absence of depressive disorder. Other variables were body mass index, appearance of any adverse event, level of physical activity per the International Physical Activity Questionnaire, ${ }^{16}$ history of depressive disorder and use of antidepressant drugs, existence of multimorbidity and/or polypharmacy, smoking and alcohol consumption (grams of alcohol per week), self-perceived health status (EuroQol EQ-5D), ${ }_{17}^{17}$ sociodemographic variables, and satisfaction with participation in the study. In addition, attendance at PA sessions for the PA group, and type of antidepressant prescribed and therapeutic compliance according to the Medication Adherence Questionnaire ${ }^{18}$ for the AT group, were assessed. The reasons envisaged for the study being brought to an end were completion of the observation period, patient dropout, or withdrawal of informed consent.

The statistical analysis strategy consisted of an initial study of the homogeneity of participant characteristics and the potentially confounding variables in both groups. We then compared the incidence of the outcome variable (MADRS score $<10)$ in both groups using the $\chi^{2}$ comparison of proportions test with a significance level of $P<.05$ and estimated the relative risks along with corresponding $95 \%$ CIs. To ascertain the trend in self-perceived health status in each group, 
we used $t$ tests of comparison of means for related samples. The efficacy analysis was performed on an intention-to-treat basis, including all patients who were initially enrolled in the study (158 in the PA group and 155 in the AT group). To ensure that the results were ascertained solely for participants who completed follow-up, we performed a per-protocol analysis for the outcome variable across the different observation periods. At the end of the observation period, we estimated the effect of the intervention on the remission of depressive symptoms using a logistic regression model, including the explanatory variables of sex, age \pm 75 years, level of physical activity, and health center where the participant was selected. Each coefficient was subjected to statistical testing using the Wald test. All analyses were performed using SPSS version 19.0 (IBM Corp).

\section{RESULTS}

The analysis included 313 participants, 158 in the PA group and 155 in the AT group, with mean (SD) baseline MADRS scores of 15.1 (4.6) and 15.9 (3.9), respectively (Table 1 ). Mean (SD) age was 72.2 (5.5) and 72.8 (6.1) years, respectively. Table 1 summarizes baseline participant characteristics, showing homogeneity between the 2 groups.

For the PA group, the mean number of sessions attended by participants was 18.9 (range 0-47; SD 16.6), with a median of 13.5 . For the AT group, the distribution of prescribed antidepressants was as follows: sertraline $26.5 \%$, trazodone $14.8 \%$, paroxetine $12.9 \%$, mirtazapine $9.0 \%$, escitalopram $8.4 \%$, venlafaxine $7.7 \%$, duloxetine $5.8 \%$, and other $14.8 \%$. In this group, $78.1 \%$ of participants displayed appropriate therapeutic compliance at 1 month.
Table 1. Baseline Characteristics of Patients in the Study Groups

\begin{tabular}{|c|c|c|c|}
\hline Characteristic & $\begin{array}{l}\text { Physical Activity } \\
\mathrm{n}=158\end{array}$ & $\begin{array}{c}\text { Antidepressant } \\
\text { Treatment } \\
n=155\end{array}$ & $\begin{array}{c}P \\
\text { Value }\end{array}$ \\
\hline \multicolumn{4}{|l|}{$\begin{array}{l}\text { Sociodemographic characteris- } \\
\text { tics, No. (\%) }\end{array}$} \\
\hline Age, y & & & .55 \\
\hline $65-74$ & $102(64.6)$ & $95(61.3)$ & \\
\hline$\geq 75$ & $56(35.4)$ & $60(38.7)$ & \\
\hline Sex & & & .437 \\
\hline Female & $127(80.4)$ & $119(76.8)$ & \\
\hline Male & $31(19.6)$ & $36(23.2)$ & \\
\hline Educational level & & & .535 \\
\hline Illiterate or primary education & $124(78.5)$ & $126(81.3)$ & \\
\hline Secondary or university education & $34(21.5)$ & $29(18.7)$ & \\
\hline Social class & & & .401 \\
\hline$|-|||^{a}$ & $23(14.6)$ & $28(18.1)$ & \\
\hline IV-Vb & $135(85.4)$ & $127(81.9)$ & \\
\hline Marital status & & & .895 \\
\hline Single, widowed, or divorced & $59(37.3)$ & $59(38.1)$ & \\
\hline Married & $99(62.7)$ & $96(61.9)$ & \\
\hline \multicolumn{4}{|l|}{ Lifestyle, No. (\%) } \\
\hline Smoker & & & .55 \\
\hline Yes & $16(10.1)$ & $19(12.3)$ & \\
\hline No & $142(89.9)$ & $136(87.7)$ & \\
\hline Alcohol consumption & & & .61 \\
\hline$>100 \mathrm{~g} /$ week & $8(5.1)$ & $6(3.9)$ & \\
\hline$<100 \mathrm{~g} /$ week & $150(94.9)$ & $149(96.1)$ & \\
\hline Physical activity & & & .412 \\
\hline Physical inactivity & $30(19.0)$ & $24(15.5)$ & \\
\hline Moderate or intense activity & $128(81.0)$ & $131(84.5)$ & \\
\hline Health status, No. (\%) & & & .707 \\
\hline Multimorbidity ( $\geq 3$ diseases) & & & .343 \\
\hline Yes & $145(91.8)$ & $144(92.9)$ & \\
\hline No & $13(8.2)$ & $11(7.1)$ & \\
\hline Polypharmacy ( $\geq 4$ drugs) & & & .501 \\
\hline Yes & $115(72.8)$ & $120(77.4)$ & \\
\hline No & $43(27.2)$ & $35(22.6)$ & \\
\hline History of depressive disorder(s) & & & .704 \\
\hline Yes & $93(58.9)$ & $97(62.6)$ & \\
\hline No & $65(41.1)$ & $58(37.4)$ & \\
\hline Prior use of antidepressant drug(s) & & & .342 \\
\hline Yes & $69(43.7)$ & $71(45.8)$ & \\
\hline No & $89(56.3)$ & $84(54.2)$ & \\
\hline Obesity & & & .465 \\
\hline Yes & $69(43.7)$ & $76(49.0)$ & \\
\hline No & $89(56.3)$ & $79(51.0)$ & \\
\hline Arterial hypertension & & & .921 \\
\hline Yes & $84(53.2)$ & $76(49.0)$ & \\
\hline No & $74(46.8)$ & $79(51.0)$ & \\
\hline & & & continues \\
\hline \multicolumn{4}{|c|}{ MADRS = Montgomery-Åsberg Depression Rating Scale. } \\
\hline \multicolumn{4}{|c|}{$\begin{array}{l}\text { a Social classes I-III: managers and university degree-related professions, administrative and service staff, } \\
\text { and self-employed workers. } \\
\text { b Social classes IV-V: skilled, semiskilled, and unskilled manual workers. }\end{array}$} \\
\hline
\end{tabular}


Table 1. Baseline Characteristics of Patients in the Study Groups continued

\begin{tabular}{|c|c|c|c|}
\hline Characteristic & $\begin{array}{l}\text { Physical Activity } \\
\mathrm{n}=158\end{array}$ & $\begin{array}{l}\text { Antidepressant } \\
\text { Treatment } \\
\mathrm{n}=155\end{array}$ & $\begin{array}{c}P \\
\text { Value }\end{array}$ \\
\hline \multicolumn{4}{|l|}{$\begin{array}{l}\text { Self-perception of health (Euro- } \\
\text { Qol-5D questionnaire), No. (\%) }\end{array}$} \\
\hline \multicolumn{3}{|l|}{ Mobility problems } & .921 \\
\hline Yes & $40(25.3)$ & $40(25.8)$ & \\
\hline No & $118(74.7)$ & $115(74.2)$ & \\
\hline \multicolumn{3}{|l|}{ Self-care problems } & .985 \\
\hline Yes & $2(1.3)$ & $2(1.3)$ & \\
\hline No & $156(98.7)$ & $153(98.7)$ & \\
\hline \multicolumn{3}{|l|}{ Problems performing daily activities } & .915 \\
\hline Yes & $20(12.7)$ & $19(12.3)$ & \\
\hline No & $138(87.3)$ & $136(87.7)$ & \\
\hline \multicolumn{3}{|l|}{ Pain or discomfort } & .89 \\
\hline Yes & $111(70.3)$ & $110(71.0)$ & \\
\hline No & $47(29.7)$ & $45(29.0)$ & \\
\hline \multicolumn{3}{|l|}{ Anxiety or depression } & .255 \\
\hline Yes & $128(81.0)$ & $133(85.8)$ & \\
\hline No & $30(19.0)$ & $22(14.2)$ & \\
\hline Health status score, mean (SD) & $59.2(18.6)$ & $57.1(16.4)$ & .287 \\
\hline MADRS score, mean (SD) & $15.1(4.6)$ & $15.9(3.9)$ & .106 \\
\hline \multicolumn{4}{|c|}{ MADRS = Montgomery-Åsberg Depression Rating Scale. } \\
\hline \multicolumn{4}{|c|}{$\begin{array}{l}\text { a Social classes I-III: managers and university degree-related professions, administrative and service staff, } \\
\text { and self-employed workers. } \\
\text { b Social classes IV-V: skilled, semiskilled, and unskilled manual workers. }\end{array}$} \\
\hline
\end{tabular}

respectively, vs AT group: $22.6 \%$ and $40.0 \%)$. However, the proportion of participants with adverse effects was significantly greater $(P=.007)$ in the AT group than the PA group. In the AT group, 22.5\% of participants reported adverse effects, with the most common being dizziness $(6.5 \%)$, epigastric pain $(5.2 \%)$, and daytime drowsiness (3.2\%). In the PA group, 14 participants $(8.9 \%)$ reported adverse effects, generally in the form of osteomuscular pain, mild contusions, or dizziness in relation to exercise. The most severe adverse effects were a fracture of the radius after a fall and fainting with spontaneous recovery.

Satisfaction with the interventions was high in both groups, with the proportion of satisfied or very satisfied participants being $87.1 \%, 97.9 \%$, and $98.5 \%$ at 1,3 , and 6 months, respectively, in the PA group, and $76.8 \%, 85.0 \%$, and $96.8 \%$ in the AT group.

On using intention-to-treat analysis, logistic regression showed

Table 2 shows the cumulative incidence of MADRS scores $<10$ over time. The intention-to-treat analysis showed that whereas this proportion was not significantly different between the PA group and AT group after 1 month, it did prove significantly different $(P<.01)$ after 3 and 6 months (PA group: $45.6 \%$ and $32.9 \%$ at 3 and 6 months, respectively vs AT group: $60.6 \%$ and $49.7 \%)$. In contrast, the perprotocol analysis showed no significant differences between the 2 groups for any of the 3 follow-up periods.

Both interventions had a favorable effect on participants' self-perceived health status (range $0-100$ points), with statistically significant $(P<.01)$ increases in both groups' health status scores over follow-up with respect to baseline, from 59.2 at the initial evaluation to 74.3 at the last evaluation in the PA group and from 57.1 to 74.3 in the AT group (Figure 2).

The proportion of withdrawals and dropouts was greater in the PA group than the AT group (PA group: $39.2 \%$ and $58.2 \%$ at 3 and 6 months, that at the end of the follow-up period, the probability of depressive symptomatology improving was greater in the AT group (odds ratio $=2.0 ; 95 \% \mathrm{CI}, 1.3-3.2$ ), without an interaction between the type of intervention undertaken and patient sex, age, initial level of physical activity, or health center where they were selected. When decrease in MADRS score was used

\begin{tabular}{|c|c|c|c|}
\hline & $\begin{array}{l}\text { Physical } \\
\text { Activity } \\
\text { n/N (\%) }\end{array}$ & $\begin{array}{c}\text { Antidepressant } \\
\text { Treatment } \\
\text { n/N (\%) }\end{array}$ & $\begin{array}{c}\text { RR } \\
(95 \% \mathrm{Cl})\end{array}$ \\
\hline & \multicolumn{3}{|c|}{1 Month } \\
\hline Intention-to-treat analysis & 76/158 (48.1) & $84 / 155(54.2)$ & $0.89(0.71-1.1)$ \\
\hline \multirow[t]{2}{*}{ Per-protocol analysis } & $76 / 135(56.3)$ & $84 / 149(56.4)$ & $0.99(0.81-1.23)$ \\
\hline & \multicolumn{3}{|c|}{3 Months } \\
\hline Intention-to-treat analysis & $72 / 158(45.6)$ & $94 / 155(60.6)$ & $0.75(0.61-0.93)$ \\
\hline \multirow[t]{2}{*}{ Per-protocol analysis } & $72 / 96(75.0)$ & $94 / 120(78.3)$ & $0.96(0.82-1.11)$ \\
\hline & \multicolumn{3}{|c|}{6 Months } \\
\hline Intention-to-treat analysis & $52 / 158(32.9)$ & $77 / 155$ (49.7) & $0.66(0.5-0.87)$ \\
\hline Per-protocol analysis & $52 / 66(78.8)$ & $77 / 93(82.8)$ & $0.95(0.81-1.11)$ \\
\hline
\end{tabular}


as the dependent variable, we found that after 6 months, there was a mean decrease of 4.04 points in the PA group and 5.8 points in the AT group, with the difference of 1.76 points (95\% CI, 0.39-3.13) between the 2 groups being statistically significant $(P=.012)$.

\section{DISCUSSION}

Our results showed that both AT and PA carried out in group sessions decreased depressive symptomatology among persons $\geq 65$ years of age diagnosed in primary care with a mild-to-moderate depressive disorder. Comparison of the 2 groups by intention-to-treat analysis showed that whereas the proportion of participants who registered improvement in their depressive symptomatology was not significantly different after 1 month, there was a significant difference at the end of 3 and 6 months. Unfortunately, attendance at the PA sessions was far less than desirable, and the number of participants who quit the study over the follow-up period was greater among those allocated to the PA group. Despite the fact that intention-to-treat analysis is the only type of analysis that conserves the advantages of randomization and maintains the comparability of both groups, it should be borne in mind that the intention-to-treat principle can underestimate the effects of an effective therapy to which adherence is poor. Analyzing the results solely for those participants who completed the follow-up (perprotocol analysis) showed that the proportion of those who experienced improvement did not differ significantly between the 2 groups at any of 3 follow-up time points. In addition, both interventions were found to be satisfactory by the participants, and both favorably affected their self-perceived health status. Lastly, the incidence of adverse effects was significantly greater in the AT group.

At present, there is overwhelming evidence attesting to the health benefits of physical exercise. ${ }^{19-21}$ Moreover, it is acknowledged that exercise can partially reverse the effects of aging of physiologic functions and preserve functional reserve in older adults ${ }^{22}$ while also acting as a protective factor and a therapeutic option for anxiety, depression, and insomnia. ${ }^{23-26}$ Ascertaining elderly patients' level of physical activity might also help identify individuals at high risk of developing depressive symptoms. ${ }^{27}$ Despite recommendations to promote exercise as a strategy to decrease the burden of chronic disease, the frequency and intensity of physical activity among people of advanced age is rather discouraging.

The results of earlier studies suggest that participation in physical exercise groups is beneficial for the treatment of depression ${ }^{28-29}$ and has the additional advantage of yielding health benefits, such as demonstrable effects on the cardiovascular and locomotor systems and socalled active aging, other than a simple improvement in depressive symptoms. ${ }^{30}$ In general, exercise brings about an improvement in the depressive symptomatology of older adults. However, studies supporting this finding have been heterogeneous and of low methodologic quality. ${ }^{31}$ A Cochrane review concluded that exercise can have a moderately beneficial effect on depressive symptoms, ${ }^{32}$ with no differences observed vis-à-vis pharmacologic treatment or psychologic therapy, though these conclusions were based on very few studies, ${ }^{33-36}$ only 1 of which was specifically conducted in individuals $\geq 65$ years of age. ${ }^{35}$ Our present study exceeds the scope of these few studies in older adults, in terms of both number of patients and duration of follow-up.

A meta-analysis showed that exercise, especially mixed aerobic and anaerobic interventions of moderate intensity, had a significant effect on depression for groups with supervised and unsupervised formats and persons presenting with no other clinical comorbidities. ${ }^{37}$ The authors suggested that the benefits of exercise have been previously underestimated owing to publication bias and that exercise should be regarded as a routine component in the management of depression in older adults. Another meta-analysis concluded that the positive effect of exercise in depressed older adults did not differ by age or type of intervention ${ }^{38}$ and that exercise is thus an effective treatment option for older adults. 
Among the limitations of the present study, we feel that the advanced age of the participants and the health problems and/or functional limitations common to older adults might have negatively influenced adherence to the 6-month PA program. When interpreting the results, account must be taken of the large number of participants lost to follow-up, especially in the PA group, something that could decisively influence the differences observed between the groups. Another important limitation that might have biased evaluation of the effects of the intervention is that this was an open trial in which the nature of the interventions ruled out the possibility of using masking techniques.

We feel that additional studies are called for that incorporate motivational strategies designed to improve older adults' adherence to exercise programs, via the inclusion of recreational activities such as arts and crafts, etc. ${ }^{39}$ More evidence is needed to show that exercise is a therapeutic resource that has indubitable health benefits and is applicable to depression, a disorder of great magnitude among older adults and one that has a great socioeconomic impact and broad-ranging effects on health-related quality of life. The additional therapeutic resource of exercise could have a favorable effect on the health and well-being of older adults and decrease health care costs. Even if an equivalence of physical exercise to pharmacologic antidepressant treatments is not established, exercise could nonetheless support pharmacologic therapy or be regarded as an alternative treatment in those cases for which pharmacologic therapy has not shown itself to be effective or is contraindicated owing to its many adverse effects and pharmacologic interactions. ${ }^{4,38}$

In conclusion, both AT and PA, carried out in group sessions, are capable of decreasing depressive symptomatology among persons aged $\geq 65$ years diagnosed in primary care with mild-to-moderate depressive disorder. Although the resulting improvement in depressive symptoms was initially similar for both treatment options, AT was superior in the medium term. Despite the fact that adverse effects were significantly greater in the AT group, both interventions were found to be satisfactory by the participants and favorably affected their self-perceived health status.

To read or post commentaries in response to this article, go to https://www.AnnFamMed.org/content/19/4/302/tab-e-letters.

Key words: depression; exercise; antidepressant agents; elderly; primary health care

Submitted December 13, 2019; submitted, revised, September 27, 2020; accepted October 29, 2020.

Funding support: This study received funding from the Carlos III Institute of Health, under Health Research Project reference number PI15/01486 (Resolution of December 16, 2015).
Previous presentation: López-Torres J, Rabanales J, Escobar F, Téllez JM, Boix C, López MA. Effectiveness of physical exercise in the treatment of depression in older adults as an alternative to antidepressant drugs [Efectividad del ejercicio físico en el tratamiento de la depresión en personas mayores como alternativa a fármacos antidepresivos]. Presented at the 36th Congress of the Spanish Society of Family and Community Medicine; June 9-11, 2016; Corunna, Spain.

Trial registration: ClinicalTrials.gov NCT03358433

Acknowledgments: The authors wish to thank the Carlos III Institute of Health - European Regional Development Fund for the financial support to undertake this study.

*DEP-EXERCISE Research Group: Luis Aguilar Salmerón (degree in Physical Activity and Sports Sciences), Clotilde Boix Gras (GP, MD), Monchi Campos Rosa (Nursing Assistant), Francisco Escobar Rabadán (GP, $\mathrm{MD})$, Concepción Escolano Vizcaíno (GP), José Luis Estellés Belenguer (GP), Juan Fernández Martín (specialist in Preventive Medicine, MD), Vicente Ferrer López (degree in Physical Activity and Sports Sciences, MD), Almudena Legido García (GP), Jaime López-Torres López (specialist in Anesthesiology and Resuscitation), Jesús López-Torres López (Pharmacist), Maria Ángeles López Verdejo (specialist in Occupational Medicine, MD), Ana López Yeste (GP), María Ángeles Lloret Callejo (Pharmacist), María Jesús Montes Lozano (specialist in Psychiatry, MD), Juana Muñoz Núñez (GP), Karen Nieto Rodríguez (specialist in Psychiatry, MD), Isabel Rodenas García (GP), Carmen Somoza Castillo (GP), Juan Manuel Téllez Lapeira (GP, MD).

\section{References}

1. Moussavi S, Chatterji S, Verdes E, Tandon A, Patel V, Ustun B. Depression, chronic diseases, and decrements in health: results from the World Health Surveys. Lancet. 2007;370(9590):851-858.

2. Dinas PC, Koutedakis $Y$, Flouris AD. Effects of exercise and physical activity on depression. Ir J Med Sci. 2011;180(2):319-325.

3. Blumenthal JA, Smith PJ, Hoffman BM. Is exercise a viable treatment for depression? ACSMs Health Fit J. 2012;16(4):14-21.

4. Kok RM, Reynolds CF III. Management of depression in older adults: a review. JAMA. 2017;317(20):2114-2122.

5. Taylor WD. Clinical practice. Depression in the elderly. N Engl J Med. 2014;371(13):1228-1236.

6. Miller KJ, Mesagno C, McLaren S, Grace F, Yates M, Gomez R. Exercise, mood, self-efficacy, and social support as predictors of depressive symptoms in older adults: direct and interaction effects. Front Psychol. 2019;10:2145.

7. Duclos M, Gouarne C, Bonnemaison D. Acute and chronic effects of exercise on tissue sensitivity to glucocorticoids. J Appl Physiol (1985). 2003;94(3):869-875.

8. Cotman CW, Berchtold NC. Exercise: a behavioral intervention to enhance brain health and plasticity. Trends Neurosci. 2002;25(6): 295-301.

9. Ernst C, Olson AK, Pinel JP, Lam RW, Christie BR. Antidepressant effects of exercise: evidence for an adult-neurogenesis hypothesis? J Psychiatry Neurosci. 2006;31(2):84-92.

10. Schuch FB, de Almeida Fleck MP. Is exercise an efficacious treatment for depression? A comment upon recent negative findings. Front Psychiatry. 2013;4:20.

11. Danielsson L, Noras AM, Waern M, Carlsson J. Exercise in the treatment of major depression: a systematic review grading the quality of evidence. Physiother Theory Pract. 2013;29(8):573-585.

12. Greer TL, Trivedi MH. Exercise in the treatment of depression. Curr Psychiatry Rep. 2009;11(6):466-472.

13. López-Torres Hidalgo J; DEP-EXERCISE Group. Effectiveness of physical exercise in the treatment of depression in older adults as an alternative to antidepressant drugs in primary care. BMC Psychiatry. 2019;19(1):21. 
14. Montgomery SA, Åsberg M. A new depression scale designed to be sensitive to change. Br J Psychiatry. 1979;134(4):382-389.

15. Lobo A, Chamorro L, Luque A, Dal-Ré R, Badia X, Baró E; Grupo de Validación en Español de Escalas Psicométricas (GVEEP). [Validation of the Spanish versions of the Montgomery-Asberg depression and Hamilton anxiety rating scales]. Med Clin (Barc). 2002;118(13):493499. [Article in Spanish]

16. Craig $\mathrm{CL}$, Marshall AL, Sjöström $M$, et al. International physical activity questionnaire: 12 -country reliability and validity. Med Sci Sports Exerc. 2003;35(8):1381-1395.

17. Rabin R, de Charro F. EQ-5D: a measure of health status from the EuroQol Group. Ann Med. 2001;33(5):337-343.

18. Morisky DE, Green LW, Levine DM. Concurrent and predictive validity of a self-reported measure of medication adherence. Med Care. 1986;24(1):67-74.

19. Blair SN. Physical inactivity: the biggest public health problem of the 21st century. Br J Sports Med. 2009;43(1):1-2.

20. Salguero A, Martínez-García R, Molinero O, Márquez S. Physical activity, quality of life and symptoms of depression in communitydwelling and institutionalized older adults. Arch Gerontol Geriatr. 2011;53(2):152-157.

21. Catalan-Matamoros D, Gomez-Conesa A, Stubbs B, Vancampfort D. Exercise improves depressive symptoms in older adults: an umbrella review of systematic reviews and meta-analyses. Psychiatry Res. 2016;244:202-209.

22. Gremeaux V, Gayda M, Lepers R, Sosner P, Juneau M, Nigam A. Exercise and longevity. Maturitas. 2012;73(4):312-317.

23. Joshi S, Mooney SJ, Kennedy GJ, et al. Beyond METs: types of physical activity and depression among older adults. Age Ageing. 2016; 45(1):103-109.

24. Garfield V, Llewellyn CH, Kumari M. The relationship between physical activity, sleep duration and depressive symptoms in older adults: The English Longitudinal Study of Ageing (ELSA). Prev Med Rep. 2016;4:512-516.

25. Aguiñaga S, Ehlers DK, Salerno EA, Fanning J, Motl RW, McAuley E. Home-based physical activity program improves depression and anxiety in older adults. J Phys Act Health. 2018;15(9):692-696.

26. de Oliveira LDSSCB, Souza EC, Rodrigues RAS, Fett CA, Piva AB. The effects of physical activity on anxiety, depression, and quality of life in elderly people living in the community. Trends Psychiatry Psychother. 2019;41(1):36-42.
27. Holmquist S, Mattsson S, Schele I, Nordström P, Nordström A. Low physical activity as a key differentiating factor in the potential highrisk profile for depressive symptoms in older adults. Depress Anxiety. 2017;34(9):817-825.

28. Sjösten N, Kivelä SL. The effects of physical exercise on depressive symptoms among the aged: a systematic review. Int J Geriatr Psychiatry. 2006;21(5):410-418.

29. Seo JY, Chao YY. Effects of exercise interventions on depressive symptoms among community-dwelling older adults in the United States: a systematic review. J Gerontol Nurs. 2018;44(3):31-38.

30. Morey MC. Physical activity and exercise in older adults. UpToDate. Accessed Nov 10, 2019. https://www.uptodate.com/contents/ physical-activity-and-exercise-in-older-adults

31. Patiño Villada FA, Arango Vélez EF, Baena LZ. Ejercicio físico y depresión en adultos mayores: una revisión sistemática. [Physical exercise and depression in the elderly: a systematic review]. Rev Colomb Psiquiatr. 2013:42(2):198-211. [Article in Spanish]

32. Cooney GM, Dwan K, Greig CA, et al. Exercise for depression. Cochrane Database Syst Rev. 2013;9:CD004366.

33. Blumenthal JA, Babyak MA, Moore KA, et al. Effects of exercise training on older patients with major depression. Arch Intern Med. 1999;159(19):2349-2356.

34. Blumenthal JA, Babyak MA, Doraiswamy PM, et al. Exercise and pharmacotherapy in the treatment of major depressive disorder. Psychosom Med. 2007;69(7):587-596.

35. Brenes GA, Williamson JD, Messier SP, et al. Treatment of minor depression in older adults: a pilot study comparing sertraline and exercise. Aging Ment Health. 2007;11(1):61-68.

36. Blumenthal JA, Sherwood A, Babyak MA, et al. Exercise and pharmacological treatment of depressive symptoms in patients with coronary heart disease: results from the UPBEAT (Understanding the Prognostic Benefits of Exercise and Antidepressant Therapy) study. J Am Coll Cardiol. 2012;60(12):1053-1063.

37. Schuch FB, Vancampfort D, Rosenbaum $S$, et al. Exercise for depression in older adults: a meta-analysis of randomized controlled trials adjusting for publication bias. Braz J Psychiatry. 2016;38(3):247-254.

38. Rhyner KT, Watts A. Exercise and depressive symptoms in older adults: a systematic meta-analytic review. J Aging Phys Act. 2016; 24(2):234-246.

39. Kim D. The effects of a combined physical activity, recreation, and art and craft program on ADL, cognition, and depression in the elderly. J Phys Ther Sci. 2017;29(4):744-747. 